\title{
PROKLA-Redaktion
}

\section{Editorial: Globalisierung des Terrors}

Die Attentate vom 11. September und die darauf folgenden Reaktionen, nicht nur der von George Bush junior ausgerufene „langanhaltende Krieg gegen den Terror", dem sich die Bundesrepublik inzwischen auch militärisch angeschlossen hat, sondern auch die verschiedenen „Sicherheitsgesetze“, die vor zukünftigen Anschlägen schützen sollen, waren der Anlass für dieses kurzfristig konzipierte Heft Globalisierung des Terrors. Der Titel ist bewusst mehrdeutig gehalten. „Terroranschläge“ sind global geworden: Selbstmordattentate, mit dem Ziel möglichst viele Menschen zu töten, finden nicht mehr nur in Israel oder Sri Lanka statt, sondern auch in den als bislang weitgehend sicher geltenden Zentren des Westens, in New York und Washington. „Terror" auf einem globalen Niveau bringen aber auch die Reaktionen auf die Anschläge hervor: die Bomben, die auf Afghanistan niedergegangen sind und dort „Kollateralschäden“ an der Zivilbevölkerung hervorgerufen haben, wurden von den betroffenen Menschen wohl kaum weniger schrecklich erfahren als von den Bürgern New Yorks die Flugzeuge, die das World Trade Center zerstörten. Und schließlich - auch dies sollte der Titel andeuten - lassen sich Attentate, wie die von New York und Washington, nicht einfach von den gegenwärtig stattfindenden Globalisierungsprozessen trennen und einem un- bestimmten Außen zuordnen, wie es die Rede vom „Bösen“ (Bush) oder vom Angriff ,auf die gesamte zivilisierte Welt" (Schröder) suggeriert.

Von der amerikanischen Regierung und den meisten Massenmedien der westlichen Welt wurden die Attentate Osama bin Laden und der Organisation AlQaida zugerechnet. Die angeblich sicheren Beweise wurden vielleicht den verbündeten Regierungen zugänglich gemacht (genaues weiß man nicht), bis jetzt aber nicht der Öffentlichkeit. Bin Laden selbst hat in verschiedenen Videos zwar nicht die Urheberschaft für die Attentate zugegeben, doch er hat sie begrüßt und mit deutlich antisemitischen Tönen zum Kampf gegen „Amerikaner und Juden" aufgerufen. Die dichotome Weltsicht des Kampfes zwischen dem Guten und dem Bösen wird offensichtlich von Bin Laden und Bush gleichermaßen geteilt. Solange Bin Laden noch Kämpfer gegen die Sowjetunion sammelte, das frühere „Reich des Bösen“, bildete diese Weltsicht eine gute Grundlage für die Zusammenarbeit von US-amerikanischen Geheimdiensten und islamischen Mudjadehin.

Als Werk „des Bösen“ sind die Attentate unfassbar. Der oft wiederholte Verweis, sie seien unvorstellbar gewesen, steht allerdings in eigentümlichen Kontrast zu den seit Jahren durchgespielten Szenarien möglicher Terrorangriffe auf die 
USA. Unerwartet war lediglich die Primitivität der Mittel: statt High-Tech Angriffen wurden simple Teppichschneider zur Kaperung von Flugzeugen benutzt, die sodann in Bomben verwandelt wurden. Diese Primitivität der Mittel kontrastiert auch mit der immer wieder erhobenen Behauptung, die Vorbereitung der Attentate habe ungeheure logistische und finanzielle Mittel erfordert - einige Tausend Dollar für Flugstunden und der Abgleich verschiedener Flugpläne reichten wohl aus. Entscheidend war nicht eine umfangreiche Logistik als vielmehr die Existenz einer Gruppe von Menschen, die bereit ist, monatelang eine Aktion vorzubereiten, die den sicheren eigenen Tod mit einschließt, was die Frage nach den gesellschaftlichen Umständen aufwirft, die eine solche Gruppe hervorbringt und die sie auf Sympathie für ihre Tat hoffen lässt.

Indem die Attentate jedoch einem von außen kommenden Bösen zugerechnet werden, erscheint die eigene Gesellschaft als gut und im Grunde gewaltfrei: Gewalttätig ist der Angreifer, die eigene Gewalt ist nur die legitime Abwehr der „freien Welt“. Die Gewalt eigener Staatlichkeit, die nicht nur historisch bei der „blut- und schmutztriefenden“ (Marx) Geburt des Kapitalismus eine Rolle spielte, sondern auch weiterhin spielt, wird damit erfolgreich ausgeblendet. Diese aus dem öffentlichen Diskurs verdrängte, historisch wie auch aktuelle Gewaltförmigkeit bürgerlicher Verhältnisse steht im Zentrum des Beitrags von Wolf-Dieter Narr. Dass die Anschläge von New York und Washington nicht unabhängig von der neoliberalen Globalisierung und der von ihr hervorgebrachten in weiten Teilen immer katastrophischere Züge annehmenden „Weltordnung“ betrachtet werden können, zeigt der Text von Joachim Hirsch auf. Sabah Alnasseri beschäftigt sich schließlich mit der Krise der arabischen Gesellschaften als Voraussetzung für ein Verständnis des Kontextes der Anschläge.

Sind die Attentate auch nicht von den ökonomischen, politischen und sozialen Verwüstungen der Globalisierung zu trennen, so macht sie dies aber noch lange nicht zu einem - wie auch immer verdrehten oder verrückten - Ausdruck des Kampfes um Befreiung. Die Flugzeuge, die das World Trade Center zerstörten und das Pentagon beschädigten, trafen nicht nur Symbole der ökonomischen und militärischen Macht der USA, sie töteten zugleich Tausende von Menschen. Diese Toten waren kein „Nebeneffekt" der Anschläge, sie müssen wohl eines ihrer Ziele gewesen sein, denn es ist nicht erkennbar, dass etwa durch eine Bombenwarnung die Zahl der Opfer hätte reduziert werden sollen. Der Massenmord an mehr oder weniger zufällig anwesenden Menschen als Ziel gab es bisher nur bei Anschlägen mit einem faschistischen Hintergrund, wie etwa dem Bombenattentat auf den Bahnhof von Bologna oder den Anschlag auf das Regierungsgebäude in Oklahoma City.

Entspringen die Attentate einem unfassbaren Bösen, sind sie nur Ausdruck eines Anschlags auf die „Zivilisation“, dann kann es nur noch um den Kampf zwischen Gut und Böse, Zivilisation und Barbarei gehen. Geht der Angriff vom „Bösen“ aus, dann steht man selbst offensichtlich auf der Seite des Guten und hat nachgerade die Pflicht, den Kampf aufzunehmen, koste es was es wolle. In den stark religiösen USA läßt sich der Krieg als „Kreuzzug“ verkaufen, und es wird von Regierungsvertretern ganz offen überlegt, dass es wohl günstiger sei, wenn man Bin Laden tot geliefert bekä- 
me, als ihn lebend gefangen zu nehmen. $\mathrm{Ob}$ der Krieg gegen Afghanistan (und seine eventuelle Fortsetzung gegen den Irak oder Somalia) tatsächlich den „Terror “" ausmerzt, lässt sich bezweifeln. Allerdings ist es der USA gelungen, unter ihrer Führung eine Allianz zu schmieden, die noch breiter ist als zu Zeiten des Kalten Krieges. Unabhängig davon, ob diese Allianz längerfristig Bestand hat, wird die USA nach dem Ende des Krieges in Afghanistan in jedem Fall im rohstoffreichen Zentralasien, wo sie bislang ohne großen Einfluß war, eine entscheidende Rolle spielen. Mit der Außenpolitik der USA, die auch nach den Attentaten mehr Kontinuitäten als Diskontinuitäten aufweist, beschäftigt sich der Artikel von Brigitte Young und Simon Hegelich.

Im säkularisierten Deutschland ist nicht von einem Kreuzzug, sondern von „Verantwortung“ und „Verpflichtung“ die Rede, der man sich nicht entziehen könne. Ganz verantwortungsbewusst beteiligt sich die rot-grüne Bundesregierung in ihrer kurzen Regierungszeit bereits am zweiten Krieg. Dass der amerikanische Verteidigungsminister erst auf deutsche Intervention hin betont, man hätte auch ein paar deutsche Soldaten angefordert (dass die USA tatsächlich auf 3.900 deutsche Soldaten angewiesen seien, ist angesichts ihrer überwältigenden Militärmaschine eine recht befremdliche Idee), macht deutlich worum es tatsächlich geht: Indem die rot-grüne Regierung ihren militärischen Beitrag der amerikanisch-britischen Allianz aufdrängt, setzt sie bruchlos den Kurs ihrer konservativen Vorgängerregierung fort, aus dem endlich vereinten Deutschland eine ganz normale Mittelmacht $\mathrm{zu}$ machen, die eben auch militärisch und nicht nur ökonomisch und politisch weltweit zum Zuge kommen will.
Auf die Attentate wurde aber nicht nur „nach außen“ mit einem Krieg gegen die „Unterstützer des Terrorismus“ reagiert, sondern auch „nach innen“ mit einem „War at Home“. Ausufernde Sicherheitsgesetze, die eine Vielzahl neuer Überwachungsmaßnahmen mit sich bringen, wurden sowohl in den USA als auch in Deutschland verabschiedet bzw. sind geplant (vgl. dazu die Beiträge von Margit Mayer/Thomas Greven und von Volker Eick/ Martin Beck/ Carsten Wiegrefe). Zwar ist bei den meisten Maßnahmen nicht zu erkennen, wie sie zur Verhinderung der Attentate vom 11. September hätten beitragen können, ihrer Zustimmung durch die Abgeordneten tat dies in einer Atmosphäre diffuser Bedrohung durch „Fremdes“ aber keinen Abbruch. Die diskursive Vermittlung solcher Bedrohungsszenarien durch die Medien, zeigt Iris Bünger anhand einer Analyse der Berichterstattung der BILD-Zeitung von September bis November.

Sowohl die US-amerikanische als auch die deutsche Wirtschaft befanden sich zum Zeitpunkte der Anschläge am Rande einer Rezession. Wie sich die wirtschaftlichen Perspektiven der USA und Deutschlands unter dem Eindruck der Anschläge darstellen, untersuchen die Beiträge von Trevor Evans und Hansjörg Herr. 